\title{
VideoNoter: A productivity tool for video data analysis
}

\author{
JEREMY ROSCHELLE and SHELLEY GOLDMAN \\ Institute for Research on Learning, Palo Alto, California
}

\begin{abstract}
The software described in this paper, VideoNoter, addresses the need for tools that support annotation and retrieval of video data and organize the presentation of multiple analyses of the same data. Video is widely perceived as an important medium for psychological research, because video recording makes the fleeting particulars of human interaction available as data for detailed analysis, while retaining much of the context of the event. Though the benefits of using video data are high, the process can be prohibitively time-consuming. We have developed a prototype computer-based video analysis tool that can enhance the productivity of the video analysis process. In this paper, we report on the design and implementation of VideoNoter, and we discuss how it facilitates video data analysis.
\end{abstract}

The software described in this paper, VideoNoter, addresses the need for tools that support annotation and retrieval of video data and that organize the presentation of multiple analyses of the same data. Video recording is an increasingly important medium for data collection in psychology and in other disciplines within the social sciences (see Byers \& Byers, 1972; McDermott, 1976; Suchman \& Trigg, 1991). Video is useful when problemsolving protocols (see, e.g., Ericsson \& Simon, 1984) or other complex behavioral data are the object of analysis, and when multiple analysts need to study and analyze the same data from different perspectives. Video is also useful for the training of junior researchers and clinicians.

With video recording, the researcher can gather diverse aspects of an event under study into a unified record, without undue cost or complications. Using video recordings as data enables the researcher to readily and repeatedly examine high-fidelity records of behavioral events. For example, video recordings simultaneously preserve vocal intonation, gestures, and gaze-features of interaction and communication that are difficult to capture through conventional observational techniques or to represent in traditional data records. The use of video also makes it possible to collect data outside of the laboratory in less controlled and structured settings, such as openended interviews or group work occurring in schools and workplaces. Because video recordings can be scrutinized repeatedly, human behavior in these more natural settings

We thank Apple Classrooms of Tomorrow, Xerox Corporation, Steelcase Corporation, National Science Foundation, and Bellcore for their support of the VideoNoter project. Randy Trigg, Steve Bagley, and Monique Barbanson contributed to the development and maintenance of the VideoNoter software. Roy Pea and our other colleagues at the Institute for Research on Learning have contributed to the design and testing of VideoNoter. Please address correspondence to Jeremy Roschelle, Institute for Research on Learning, 2550 Hanover Street, Palo Alto, CA 94304. can support extended analysis, and coding and observation schemes can be developed that incorporate more details of the contents and the contexts of behavior.

Yet despite these advantages, working with video media has been cumbersome and time-consuming in comparison with the use of more conventional media, such as transcripts and coded databases. One problem with video involves the annotation of data. Transcripts and coding conventions are typically used to represent behavior. It is easy to add comments and codes to a transcript, typically by writing in the margin, and these annotations can have the convenient property of always appearing next to the transcript to which they refer. By contrast, there have been no simple ways to link annotations to video segments, and it is difficult to keep the original video data available to the analyst once notes and transcripts have been developed. A second problem regards the retrieval of coded data segments. It has been much faster to find a particular place in a transcript than it has in a video. If the transcript is on a computer, for example, a word search can find particular places almost instantaneously. Finding a particular utterance or sequence on a videotape has been both a time-consuming and a frustrating process.

Even when video records of events are the primary source of data, the difficulties with annotation and retrieval have forced most researchers to proceed by working with secondary data such as transcripts or codes, rather than analyzing the video itself. The video record is used only once, to produce the transcript or codes on which the analysis is based. In some areas of study, this loss of a generation of data is consequential for the ability of the analyst to verify particular patterns in the data and to present results to other researchers.

Computer software can automate the process of accessing and retrieving selected segments of video data. With VideoNoter, the primary data record is linked with the secondary records such as transcripts, codes, and annotations. Moreover, particular frames and segments of 
video data can be located and retrieved quickly. In addition, multiple analyses of the same data can be examined side by side while one consults the primary video data. The issue of organizing presentations of multiple streams of analysis frequently arises in multidisciplinary collaborations. Video data supports multidisciplinary research because it provides a rich, yet unified record of a particular behavioral event. Thus each team member can focus on particular aspects of a video segment, while staying grounded in the same primary data. Relating and synthesizing multiple perspectives on the same data are essential if multidisciplinary work is to proceed.

Other software projects have addressed aspects of the video data analysis process. For example, some researchers (e.g., O. Barnes, Haith, \& Roberts, 1988; Ergener \& Wellens, 1989) have developed methods of logging behavioral events (e.g., keypresses) directly onto videotape. Krauss, Morrel-Samuels, and Hochberg (1988) discuss a tool for coding and analyzing streams of events recorded on videotape. H. J. Barnes, Vaughn, Jorgensen, and Rosenbaum (1989) present a software and hardware system for coding nonverbal behaviors. VideoNoter complements these tools by facilitating interactive annotation and analysis of video data. It addresses the three issues outlined above: (1) the linking of transcripts and analytic codes to video, (2) the retrieving of segments of video quickly and reliably, and (3) the integration of multiple analyses of the same data.

\section{DESCRIPTION OF VIDEONOTER}

\section{VideoNoter Hardware}

VideoNoter runs on any Apple Macintosh computer with a hard disk and 4 MB of RAM. It works with a variety of video playback devices. These devices differ in cost, as well as retrieval speed and accuracy. We mainly use three devices (see Table 1). A consumer VCR is most cost-effective, but access is slow and inaccurate. A professional VCR can achieve perfect accuracy ("frame accuracy"), but at a greater cost. A video laser disk player optimizes speed and accuracy, although the cost of producing laser disks can be unacceptably high for many applications.

\section{VideoNoter Software}

The researcher who uses VideoNoter types annotations on the computer on a form called a "worksheet" (see Figure 1). Worksheets are divided into a set of columns called "panes." One pane (the leftmost column in Figure 1) displays a time line. This time line corresponds to the du- ration of the video data. In addition, a gray bar marks a specific place in the time line (1:04:32 in Figure 1). The computer constantly updates the gray bar so that it shows the location in the video that is currently visible on the TV monitor. When the video is playing, for example, the bar moves down the time line; when the video is rewinding, the bar moves up the time line.

In the panes next to the time line, the researcher can type comments or transcripts, fill out coding forms, or place graphics. These annotations automatically appear in a position in the pane corresponding to the place in the time line that they refer to. In Figure 1, " $\mathrm{S} 1$ focuses attention" refers to an event that occurred at approximately 1:04:22.

The researcher may create any number of panes. Each pane can display different kinds of data, such as transcripts, coding forms, graphics, digitized video pictures, written comments, or logs of event data from another device (e.g., an eye-tracking machine). Multiple perspectives are typically displayed by devoting one pane to each perspective. If there are more panes than fit on the computer screen at one time, the analyst can chose a subset of panes to view.

The user can control the video player from either the keyboard or a menu. The available commands include all standard video player operations: play, pause, rewind, and so forth. The use of keyboard or menu commands is especially convenient for transcription and coding-the researcher can play, stop, and review the video without lifting fingers off of the keyboard.

VideoNoter automates the process of locating a particular segment of video. To retrieve a segment of video, the user drags the gray bar with the mouse to the desired place in the time line. The computer then calculates the video player operations necessary to go from the current location to the desired location, and issues the commands to the video player. With this feature, the user can easily command the video player to go to an exact time, like $3: 12: 32$. Moreover, one can easily see the segment of video that corresponds to a particular annotation. For example, to see the segment of video on which the subject says "That was the black arrow" in Figure 1, the researcher would click the mouse at a location in the time line next to the annotation. VideoNoter would then move the video to the desired location. Response time for a videodisk is quick $(<2 \mathrm{sec})$. Response time for a videotape depends on the distance between the current and desired locations. But since the search process does not require the user's attention once the search command has been issued, the user can work on something else while

Table 1

Types of Video Players Compatible with VideoNoter

\begin{tabular}{lcccc}
\hline & Player cost & Media cost & Retrieval & Accuracy \\
\hline Consumer VCR & $\cong \$ 500$ & $\$ 0.10 / \mathrm{min}$ & very slow & $\pm 2 \mathrm{sec}$ \\
Professional VCR & $\cong \$ 5,000$ & $\$ 0.10 / \mathrm{min}$ & slow & $1 / 30 \mathrm{sec}$ \\
Laser disk & $\cong \$ 1,000$ & $\$ 10.00 / \mathrm{min}$ & very fast & $1 / 30 \mathrm{sec}$ \\
\hline
\end{tabular}




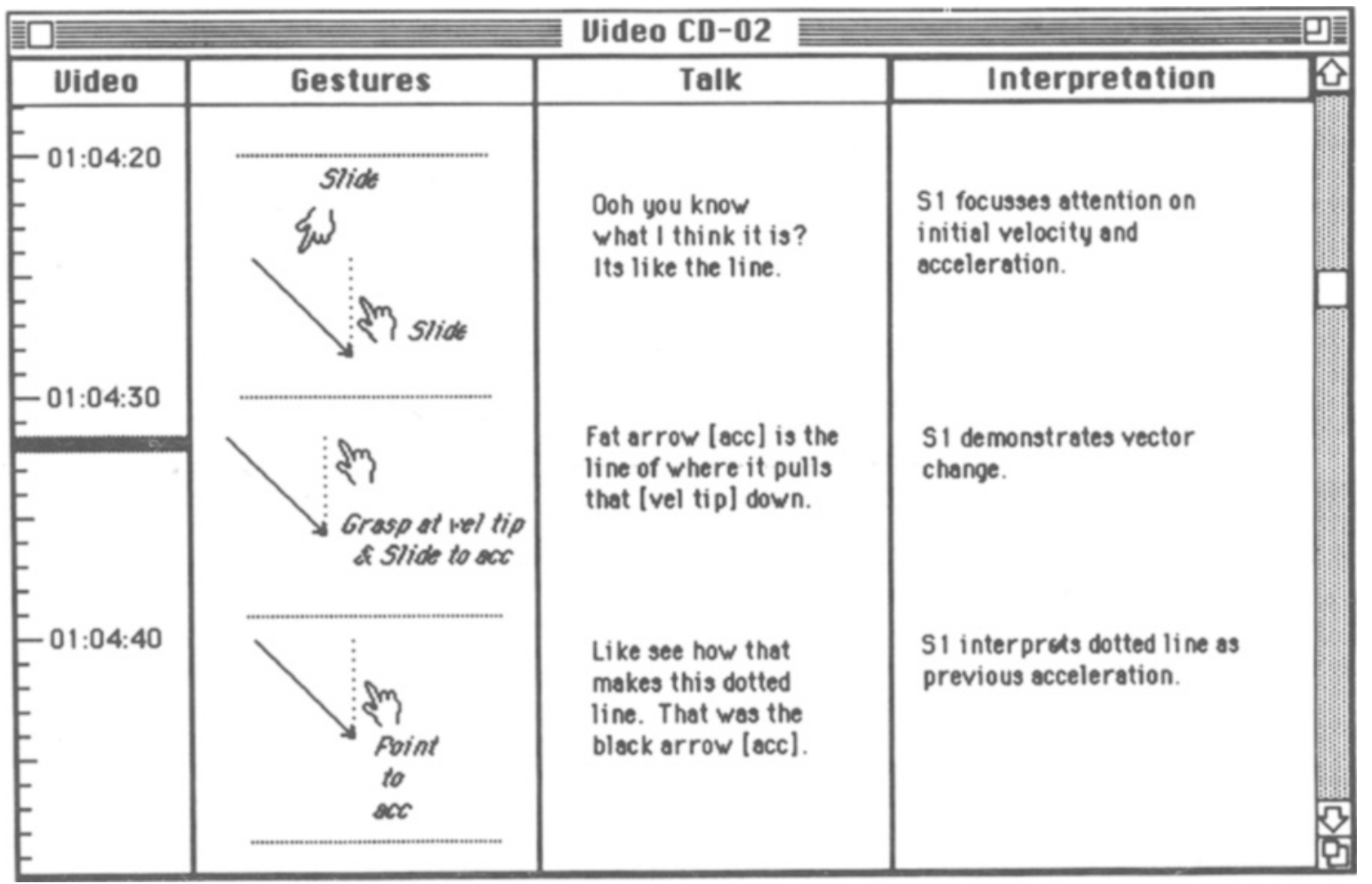

Figure 1. A VideoNoter worksheet.

VideoNoter moves the tape to the desired location. In contrast, without VideoNoter, the user must guess how long the fast forward or rewind button should be held down to arrive at the desired time and must attentively watch the machine as the video nears the desired point.

VideoNoter has a number of other features, including means for grouping related video segments in a "collection window." These additional features, as well as technical specifications, are discussed in Roschelle, Pea, and Trigg (1990).

\section{USING VIDEONOTER}

\section{Progressive Refinement}

Our experience with VideoNoter suggests that it can enhance the productivity of psychological research by enabling that research to proceed via a progressive refinement process. By progressive refinement, we mean starting with a coarse coding of the entire video, and gradually adding layers of more detailed annotations that are selectively focused on appropriate segments of data. Progressive refinement can be a productive research strategy, because it focuses labor-intensive tasks on the most important sections of data. Here, we will illustrate the productivity enhancements made possible by VideoNoter with an example from one of our projects. This project is one of several at the Institute for Research on Learning in which VideoNoter is currently being used. It involves an investigation of the process of collaborative problemsolving in a computer-based learning task (Roschelle, 1991).

The project involves 20 videotapes, each one of which is approximately $90 \mathrm{~min}$ long, which together add up to a total of about $30 \mathrm{~h}$ of tape. The contents of the tapes have similar structures: each tape covers a session with a student dyad, and each session consists of an introduction, 10 tasks, and a structured interview. The tasks, however, have a different order on every tape, and they vary in length. Moreover, as is typical with video data, our tapes are dense with verbal and nonverbal communication, as well as task-specific behaviors. Good transcripts of the tapes require 50-100 h of transcription work per hour of tape, making transcription a significant impediment to productivity.

Instead of turning to transcription immediately, we adopted a strategy of progressive refinement in which we made a series of passes through the videotapes, each time focusing on smaller samples of tape in greater detail. Figure 2 shows annotation panes corresponding to three successive passes through the data. The leftmost pane was an ad hoc content log. In this pane, the researcher typed descriptions of events that might be important during the later analysis, such as the students' focus of attention, problem-solving routines, and problem-solving strategies. Later, the researcher coded the students' problem-solving. Codes are entered through the use of templates, such as 


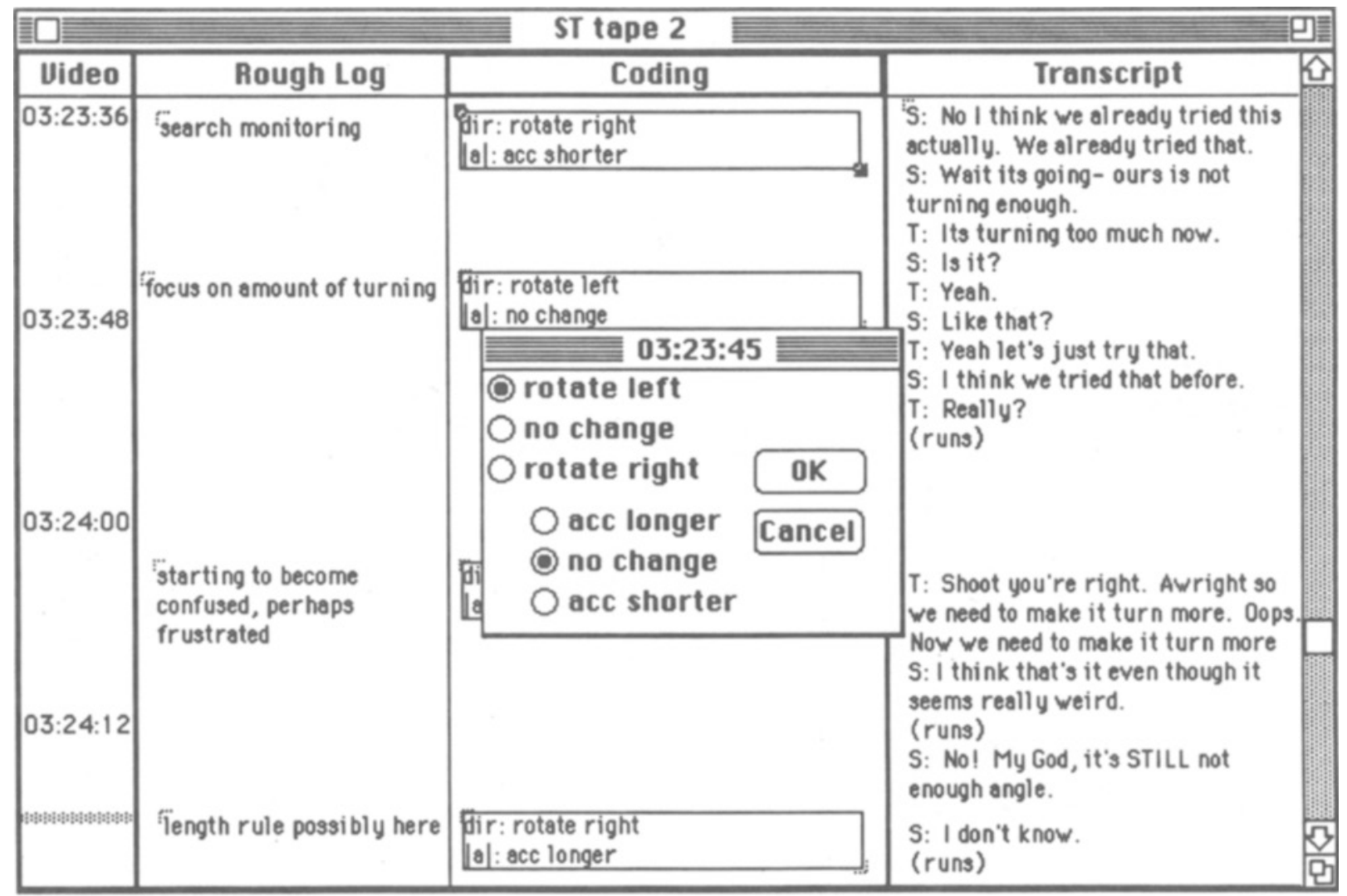

Figure 2. An example of progressive refinement.

the one labeled " $03: 23: 45$ " in Figure 2. In this particular task, the moves consisted of making an arrow longer or shorter, and rotating left or right. Coded sequences of moves were used to compare the students' problemsolving with a model. In a third pass, the researcher transcribed the students' conversation, as shown in the rightmost pane.

Each layer of refinement in our annotation introduced a more detailed research focus. For example, on the basis of the content logs, we identified a few of the tasks as being more interesting than the others. These interesting tasks were coded first. The coded data was then compared with our problem-solving model. Figure 2 shows the worksheet for two students named "Scott" and "Tom." Because Scott and Tom's moves on this task did not match the model well, the researcher added an additional layer of annotation, a transcript of their conversation. By analyzing Scott and Tom's moves in conjunction with their conversation, we were able to account for their divergence from the problem-solving model. Through a progressive refinement approach, we were able to code the data selectively and to transcribe the video recording even more selectively. This enabled us to proceed to the heart of our data more quickly, and to avoid wasting unnecessary time and expense in coding behaviors and transcribing conversations.
Moreover, we found that VideoNoter changed the nature of the analysis process substantially. For example, VideoNoter made it easy to find locations of the certain tasks in each of our tapes, even though the timings of the tasks were random. Thus, we could rapidly compare the videotapes of all the different students working on the task. For example, at one point, we wanted to know whether other students exhibited the kinds of behaviors Scott and Tom did on this particular task. With VideoNoter, we were able to rapidly find and watch the appropriate segments on each of 13 additional tapes. Within a couple of hours, we were able to ascertain that Scott and Tom's behaviors were unique. Without VideoNoter, we would have had to dedicate substantial preparation time before embarking on such a comparison.

Similarly, we used VideoNoter's search capabilities to pull up all annotations related to a theme and then watch all the video segments relevant to that theme. This made it easy to track a certain theme throughout a tape, watching only the segments relevant to the theme and skipping over irrelevant material. For example, the content log of Scott and Tom's performance contains a reference to a particular problem-solving rule. As part of one analysis, we searched for all occurrences of this rule in the content $\log$ and used VideoNoter to retrieve the corresponding segments of video. Watching this video enabled us 
to observe how Scott and Tom modified their application of the rule over time.

The same process was useful for tracking the consistency of our coding, for we could skip quickly to several events that had been assigned identical codes and decide whether or not they were in fact similar events. (Such skipping around is particularly fruitful on videodisk, because videodisk players take only a few seconds to skip to any location, allowing the analyst to perceive true juxtaposition of the segments.) In addition, VideoNoter's organization of annotations into panes helped us maintain a coherent view of our annotations-rough content logs, coded moves, and transcripts relating to the same segment of video were viewed side by side in parallel panes. This spatial organization of time in the vertical dimension and of annotation types in the horizontal dimension presented a comprehensible view of a multifaceted analysis.

\section{Multidisciplinary Research}

VideoNoter is designed to address issues arising when multiple analyses of the same data need to be related. These issues are particularly salient in interdisciplinary collaborations. Because video provides a diverse, yet unified, record of behavioral events, each team member can focus on particular aspects of a video segment while staying grounded in the same event. We use the Dynagrams Project to illustrate one way in which VideoNoter aided us in our interdisciplinary research at the Institute for Research on Learning.

The Dynagrams project team consisted of members from the fields of psychology, science education, interface design, and educational anthropology. In this project, we examined how students learn to construct, use, and comprehend scientific diagrams. A software program was constructed that allows students to build and model diagrams of geometric optics phenomena. The overall research plan called for an examination of existing patterns of classroom teaching and learning with diagrams, along with a study of the role of the Dynagrams software in changing those patterns (Pea, in press). When Dynagrams was field-tested in a high school physics classroom, the project team observed two periods of lessons daily and made video recordings of the teachers and selected student work groups.

Each team member came to the data analysis with particular kinds of questions and methods for treating data analysis. The psychologist examined students' problemsolving and explanatory behaviors in relation to their use of diagrams. The educational anthropologist investigated how the students used the diagrams to further their collaborations in problem-solving and learning. The science education specialists traced students' progress at learning the subject matter. The software developers analyzed difficulties that students encountered in using the Dynagrams software. These concerns required each team member to study and analyze the classroom video records in particular kinds of ways. As each researcher focused on his or her own concerns, the complementary findings of others influenced the analyses. For example, the software developers needed to connect their analyses of the students' interaction with the software with the cognitive, collaborative, and subject-matter views in order to determine how best to refine the technology.

VideoNoter was particularly helpful for organizing the preliminary treatments of the data, and for providing a format for cross-disciplinary work that allowed for the verification of conjectures with the primary record of the events. In particular, the team benefited from early refinements of data made possible by VideoNoter. Content logs were made of $200 \mathrm{~h}$ of classroom tape and $30 \mathrm{~h}$ of student interviews. The content logs made it possible to identify $30 \mathrm{~h}$ of group work around the simulator for transcription and further analysis by team members. A worksheet for each of those tapes was created with separate panes containing content logs and transcripts. The ability to "hide" panes made it possible for two or more of the team members to analyze the same behavior separately and then compare their observations and interpretations. These exercises helped establish practices for the identification of critical aspects of the analyses while team members refined their interpretations. Team members also created panes for each worksheet that contained their annotations of the video record. These panes were referred to as members worked independently on their analyses, as well as during whole team sessions. For example, when the anthropologist identified a collaborative process by means of which students voiced and evaluated their conjectures about how light refracts, she referred to the annotations by the physics educator to confirm her hunch that the students were grappling with a new idea. This sort of triangulation among multiple views of the primary behavioral record enabled the team to progress toward the overall project goal despite the differences among the particular analytic perspectives.

\section{Presentations and Training}

VideoNoter is also a powerful tool for preparing presentations and for training new members of a research team. It enables the researcher to demonstrate quickly how analytic categories are connected to observable behaviors. In the case of presentations, video gives the audience an opportunity to examine the validity, as well as the internal consistency, of the analysis. In the case of training, video gives the trainee a chance to become oriented to the actual events that the trainee will be annotating, rather than to a merely linguistic description of the events.

We have used VideoNoter to assemble data for both formal and informal presentations. An example of a formal presentation is a recent National Science Foundation project review of Dynagrams. For such a review, it is standard to show the software that was developed, and then to present analyses of the changes in students' learning as a function of using the software. In addition, we 
used VideoNoter to retrieve a set of video segments that showed students using the software. This enabled the reviewers not only to see our design and its outcomes, but also to see how the software supported students' learning.

Less formal presentations occur routinely when visitors come to our laboratory. A group of Soviet psychologists visited recently; they were particularly interested in the theme of how diagrams mediate human interaction. Although there was a severe language barrier, we sparked a provocative and productive discussion by using VideoNoter to quickly retrieve a set of video segments relevant to the Soviets' interests. By focusing on the students' nonverbal behavior on the videotape, we overcame the barriers to a serious discussion of our research.

We also use VideoNoter to train new members of research teams. New members can begin by coding video data from our archives. By hiding and showing the existing annotations to the video, trainees can compare their coded data to our standards, and thereby learn how we code data.

\section{CONCLUSION}

The use of video as data can enhance the research process by making detailed records of behavioral events available for repeated inspection and analysis. Current technology offers cost-effective, high-quality cameras for making video recordings. Moreover, computer database and statistical packages offer good tools for storing observations and analyzing patterns. VideoNoter complements these tools by (1) linking annotations to video data, (2) providing fast, reliable, and accurate retrieval of marked video segments, and (3) organizing multifaceted analyses in multiple columns. VideoNoter facilitates a progressive refinement approach to data analysis, supports multidisciplinary project teams, and provides assistance for presentations and training.
VideoNoter is presently under development at the Institute for Research on Learning. It is not yet available to the general public.

\section{REFERENCES}

Barnes, H. J., Vaughn, J., Jorgensen, M. J., \& Rosenbaum, D. A. (1989). A low-cost method for digitizing videotaped continuous movements on the Macintosh. Behavior Research Methods, Instruments, \& Computers, 21, 255-258.

Barnes, O., Haith, M. H., \& Roberts, R. J., JR. (1988). Simultaneous electronic recording of video and digital information on the video channel of a VTR or VCR. Behavior Research Methods, Instruments, \& Computers, 20, 32-36.

BYERS, P., \& BYERS, H. (1972). Non-verbal communication and the education of children. In C. Cazden, V. John, \& D. Hymes (Eds.), Functions of language in the classroom (pp. 3-31). New York: Teachers College Press, Columbia University.

ERgener, D., WELLENS, A. R. (1989). DataVision: A computerbased system for generating and reading digital information accompanying video images on videotape. Behavior Research Methods, Instruments, \& Computers, 21, 401-407.

Ericsson, K. A., Simon, H. A. (1984). Protocol analysis: Verbal reports as data. Cambridge, MA: MIT Press.

Krauss, R. M., Morrel-Samuels, P., \&ochberg, J. (1988). VIDEOLOGGER: A computerized multichannel event recorder for analyzing videotapes. Behavior Research Methods, Instruments, \& Computers, 20, 37-40.

MCDERMOTT, R. P. (1976). Kids make sense: An ethnographic account of the institutional management of success and failure in one first grade classroom. Unpublished doctoral dissertation, Stanford University.

PEA, R. D. (in press). Augmenting the discourse of learning with computer-based learning environments. In E. De Corte, M. Linn, H. Mandl, \& L. Verschaffel (Eds.), Computer-based learning environments and problem solving (Proceedings of NATO ARW). New York: Springer.

Roschelle, J., Pea, R., Trigg, R. (1990). VideoNoter: A tool for exploratory video analysis (IRL Report No. 90-0021). Palo Alto, CA: Institute for Research on Leaming.

ROSCHELLE, J. (1991). The structure of qualitative knowledge about velocity and acceleration. Manuscript in preparation.

Suchman, L., TrigG, R. (1991). Understanding practice: Video as a medium for reflection and design. In J. Greenbaum \& M. Kyng, Designing by doing: $A$ tool box approach to collaborative system design (pp. 66-89). Hillsdale, NJ: Erlbaum. 